\title{
A compound heterozygote of novel and recurrent DTDST mutations results in a novel intermediate phenotype of Desbuquois dysplasia, diastrophic dysplasia, and recessive form of multiple epiphyseal dysplasia
}

\author{
Atsushi Miyake · Gen Nishimura · Toru Futami • \\ Hirofumi Ohashi $\cdot$ Kazuhiro Chiba $\cdot$ Yoshiaki Toyama • \\ Tatsuya Furuichi $\cdot$ Shiro Ikegawa \\ Received: 27 March 2008/Accepted: 19 May 2008/Published online: 14 June 2008 \\ (C) The Japan Society of Human Genetics and Springer 2008
}

\begin{abstract}
Diastrophic dysplasia sulfate transporter (DTDST) is required for synthesis of sulfated proteoglycans in cartilage, and its loss-of-function mutations result in recessively inherited chondrodysplasias. The 40 or so DTDST mutations reported to date cause a group of disorders termed the diastrophic dysplasia (DTD) group. The group ranges from the mildest recessive form of multiple epiphyseal dysplasia (r-MED) through the most common DTD to perinatally lethal atelosteogenesis type II and achondrogenesis 1B. Furthermore, the relationship between DTDST mutations, their sulfate transport function, and disease phenotypes has been described. Here we report a girl with DTDST mutations: a compound heterozygote of a novel p.T266I mutation and a recurrent p. $\Delta$ V340 mutation
\end{abstract}

\author{
A. Miyake $\cdot$ T. Furuichi $\cdot$ S. Ikegawa $(\bowtie)$ \\ Laboratory for Bone and Joint Diseases, \\ Center for Genomic Medicine, RIKEN, 4-6-1 Shirokanedai, \\ Minato-ku, Tokyo 108-8639, Japan \\ e-mail: sikegawa@ims.u-tokyo.ac.jp \\ G. Nishimura \\ Department of Radiology, Tokyo Metropolitan Kiyose \\ Children's Hospital, Kiyose, Tokyo, Japan \\ T. Futami \\ Department of Orthopaedic Surgery, \\ Shiga Medical Center for Children, \\ Moriyama, Shiga, Japan \\ H. Ohashi \\ Department of Heredity Clinic, \\ Saitama Children's Medical Center, \\ Saitama, Japan \\ A. Miyake · K. Chiba · Y. Toyama \\ Department of Orthopaedic Surgery, \\ Keio University School \\ of Medicine, Shinjuku, Tokyo, Japan
}

commonly found in severe phenotypes of the DTD group. In infancy, the girl presented with skeletal manifestations reminiscent of Desbuquois dysplasia, another recessively inherited chondrodysplasia, the mutations of which have never been identified. Her phenotype evolved with age into an intermediate phenotype between r-MED and DTD. Considering her clinical phenotypes and known phenotypes of p. $\Delta \mathrm{V} 340$, p.T266I was predicted to be responsible for mild phenotypes of the DTD group. Our results further extend the phenotypic spectrum of DTDST mutations, adding Desbuquois dysplasia to the list of differential diagnosis of the DTD group.

Keywords Diastrophic dysplasia sulfate transporter (DTDST) · Diastrophic dysplasia (DTD) - Recessive form of multiple epiphyseal dysplasia (r-MED) - Desbuquois dysplasia $\cdot$ Genotype-phenotype correlation

\section{Introduction}

The diastrophic dysplasia sulfate transporter gene (DTDST, alias $S L C 26 A 2)$ encodes membrane protein with 12 transmembrane domains composed of 739 amino acids. DTDST transports sulfate and contributes to synthesis of sulfated proteoglycans in cartilage. Approximately 40 DTDST mutations have been reported (Rossi and Superti-Furga 2001) in four autosomal recessive chondrodysplasias, including two nonlethal disorders, a recessive form of multiple epiphyseal dysplasia (r-MED) (Superti-Furga et al. 1999), and diastrophic dysplasia (DTD) (Hastbacka et al. 1994); and two lethal disorders, atelosteogenesis type II (AO-II) (Hastbacka et al. 1996) and achondrogenesis 1B (ACG-1B) (Superti-Furga et al. 1996a). These disorders constitute a disease spectrum termed the diastrophic 
dysplasia (DTD) group (Lachman 1998; Hall 2002; Superti-Furga et al. 2007).

DTDST mutations result in reduction in either sulfate uptake or proteoglycan sulfation. In fact, chondrocytes and cartilage matrices with chondrodysplasias with DTDST mutations show a deficiency of intracellular sulfate and extracellular proteoglycan (Hastbacka et al. 1996; Supertifurga et al. 1996a; Rossi et al. 1997, 1998). Investigation for sulfate transporter function and cell localization of mutant DTDSTs have revealed that DTDST mutations are classifiable into partial-function mutations and null mutations (Karniski 2001, 2004; Maeda et al. 2006). Partialfunction mutants have 39-69\% sulfate transport activity compared with the wild-type DTDST (Karniski 2004). These mutant proteins are properly expressed on the cell membrane, but they are significantly less than the wild-type protein. Null mutations including p. $\Delta$ V340 create trace amounts of proteins either from poor expression or from rapid degradation. These proteins are expressed only intracellularly, not on the plasma membrane (Karniski 2004).

The genotype-phenotype correlation of DTDST mutations has been well described (Superti-Furga et al. 1996b; Rossi and Superti-Furga 2001; Karniski 2001, 2004). The current concept includes homozygotes for null mutations resulting in ACG-1B, heterozygotes for both null and partial-function mutations in either AO-II or DTD, and homozygotes for partial-function mutations in r-MED. However, some variants of these diseases, such as McAlister dysplasia as a variant of AO-II (Rossi et al. 1997) and broad bone platyspondyly as a variant of DTD (Mégarbané et al. 1999), have also been described. Thus, the spectrum of phenotypes caused by DTDST mutations may extend further.

Desbuquois dysplasia is a rare, nonlethal, autosomal recessive disease, and its causative gene was hitherto unknown. Desbuquois dysplasia is characterized by marked short stature of prenatal onset, joint laxity, round face, bulging eyes, midface hypoplasia, "Swedish-key" appearance of the proximal femora, hyperphalangy of the index finger, and advanced carpal and tarsal bone ossification (Faivre et al. 2004). The phenotypic variations are diverse, and mild Desbuquois dysplasia without hyperphalangy has been reported (Nishimura et al. 1999). The phenotype variations cause diagnostic confusion, and Desbuquois dysplasia is occasionally misdiagnosed as other chondrodysplasias such as Larsen syndrome.

Here we report an unusual phenotype in a compound heterozygote of a novel p.T266I mutation and a common p. $\Delta$ V340 mutation. The phenotype was indistinguishable in infancy from that of Desbuquois dysplasia, but it evolved into an intermediate between r-MED and DTD. Our experience raises the possibility of a novel phenotype generated by a novel DTDST mutation and implies difficulty in differential diagnosis between Desbuquois dysplasia and mild phenotypes in the DTD group on clinical and radiological grounds.

\section{Materials and methods}

\section{Clinical report}

The girl was born to a healthy nonconsanguineous Japanese couple by normal delivery at 40 weeks gestation. Her birth height was $49.5 \mathrm{~cm}$. She had bilateral clubfeet, contracture of the MP joints, and hyperextension of bilateral knees. She also had short limbs involving all segments. The midface was somewhat flattened. Radiographs at 2 weeks of age revealed mildly broadened long bones of the legs, bilateral hip subluxation, and bilateral reduction in the talocalcaneal angles of the feet. Radiographs at 7 months of age showed broadening and Swedish-key appearance of the proximal femora and advanced carpal bone ossification (Fig. 1), which led to a diagnosis of Desbuquois dysplasia. Short stature became obvious at 3 years of age and was remarkable at 4 years of age $(88 \mathrm{~cm}$ : $-3 \mathrm{SD})$. On radiological examination at 4 years of age, our attention was drawn to proximal femoral epiphyseal dysplasia and broadening of the short tubular bones, resembling those of r-MED (Fig. 2). The findings led us to a molecular analysis of the DTDST.

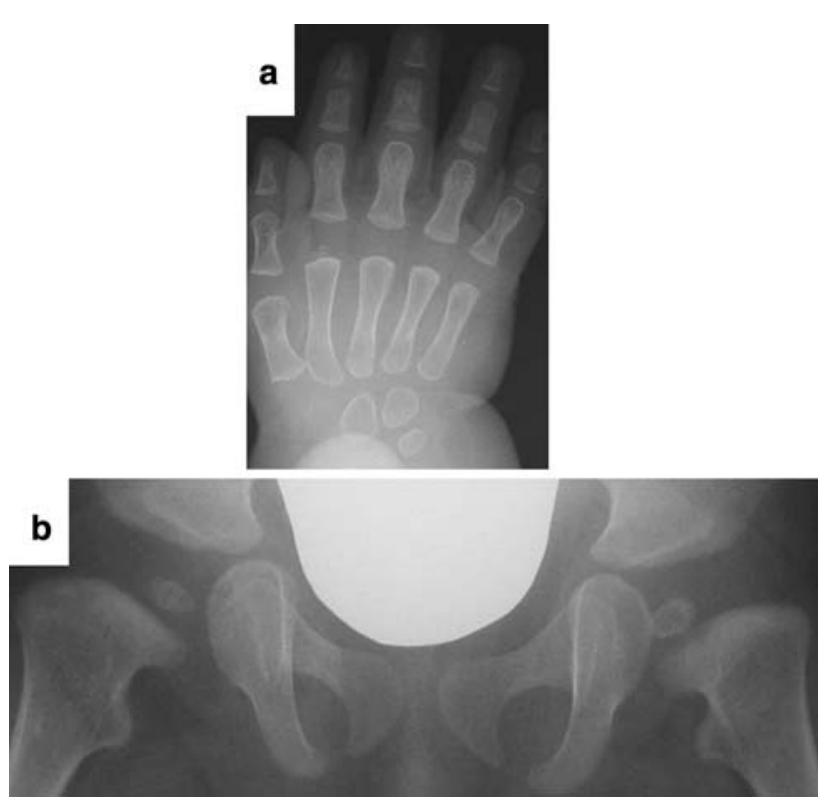

Fig. 1 X-rays at 7 months of age. a Metacarpal bones and phalanges were broad. Four carpal bones were visible, indicating that carpal bone ossification was advanced. b Swedish-key appearance of proximal femora were visible 


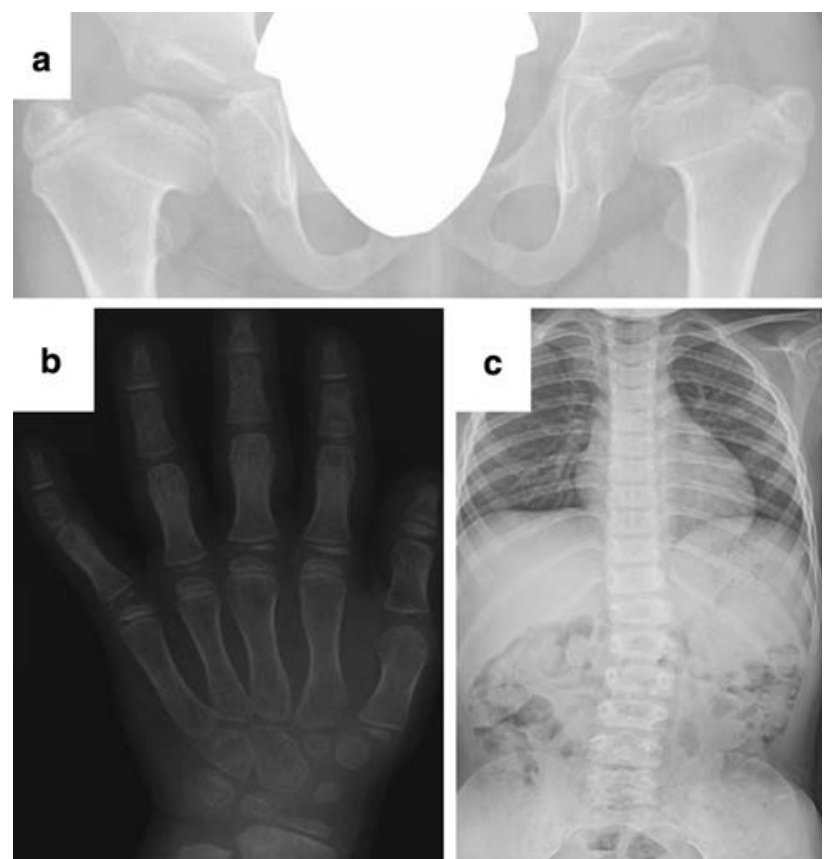

Fig. 2 X-rays at 4 years of age. a Bilateral hip joints had acetabular dysplasia. Femoral heads were slightly flat and femoral necks were broad. As a result, coxa vara was displayed. b Carpal bone ossification was advanced; seven carpal bones were visible in this 4-year-old hand. c Total spine showed mild scoliosis and mild flattening of vertebrae

\section{Detection of mutations}

Peripheral blood samples of the patient, her parents, and 96 unrelated normal Japanese controls were obtained with written informed consent. Genomic deoxyribonucleic acid (DNA) samples were extracted by standard procedures. The entire coding region and flanking intronic regions were examined by polymerase chain reaction (PCR) and direct sequence analysis (Ikeda et al. 2001). Direct sequencing was performed using an $\mathrm{ABI}$ prism 3700 automated sequencer (Applied Biosystems, Foster City, CA, USA). To obtain allelic information, PCR products containing mutations were cloned using a TOPO TA cloning kit (Invitrogen, Carlsbad, CA, USA) and sequenced. The paternity was confirmed by ten unlinked microsatellite markers. They were genotyped using an ABI prism linkage mapping set v2.5 and an ABI prism 3700 automated sequencer (Applied Biosystems) by standard procedures.

\section{Evaluation of mutations}

To investigate the conservation of mutated amino acids p.T266 and p.V340, reference sequences of human (NP_000103), horse (NP_001075403), mouse (NP_031911),
Table 1 A summary of mutations in this case

\begin{tabular}{lllll}
\hline Nucleotide & Amino acid & dbSNP No & AF & Mutation DB \\
\hline c.797C $>$ T & p.T266I & $(-)$ & $(-)$ & $(-)$ \\
c.1018-1020del & p.4V340 & $(-)$ & $(-)$ & ACG1B, DTD \\
c.2065A $>$ T & p.T689S & rs3776070 & 0.167 & Polymorphism
\end{tabular}

p. $\Delta$ V340: a common mutation reported as null mutation (Karniski 2004)

$A C G 1 B$ achondrogenesis $1 \mathrm{~B}, D T D$ diastrophic dysplasia, $A F$ allele frequency, (-) no data in database

chicken (XP_425183), and zebra fish (XP_685114) were obtained from the National Center for Biotechnology Information (http://www.ncbi.nlm.nih.gov/). PSORT, a computer program for the prediction of protein localization sites in cells (http://psort.nibb.ac.jp/), was used to predict the structure before and after introduction of p.T266I.

\section{Results}

\section{Detection of mutations}

The entire coding region and flanking intronic regions of DTDST were directly sequenced. Two heterozygous mutations, c.797C $>\mathrm{T}$ (p.T266I) and c.1018-1020del (p. $\Delta$ V340), were detected (Table 1). The former was not found in the public database for single nucleotide polymorphism (SNP) and mutation or in 96 unrelated Japanese controls, whereas the latter was recurrent (Superti-Furga et al. 1996b). Polymerase chain reaction (PCR) products containing two mutations were cloned to obtain allelic information, and the patient was recognized as a compound heterozygote of the two mutations (Fig. 3a). The two mutations were searched in the parents by direct sequence. A heterozygous mutation of c.1018-1020del (p. $\Delta$ V340) was recognized in the mother, but neither of the mutations was found in the father (Fig. 3b). Paternity was confirmed by genotyping unlinked ten microsatellite markers for the patient and parents.

\section{Evaluation of mutations}

Amino acid sequences of DTDST were compared between five diverse species; p.T266 and p.V340 were conserved in all five species (Fig. 4a). Using PSORT, the structural change of DTDST protein was predicted. T266 was outside of the cell, but I266 was in the plasma membrane. The introduction of p.T266I caused ten-amino-acids shortening of the third cytoplasmic domain and ten-amino-acids elongation of the third extracellular domain of the DTDST protein (Fig. 4b). 


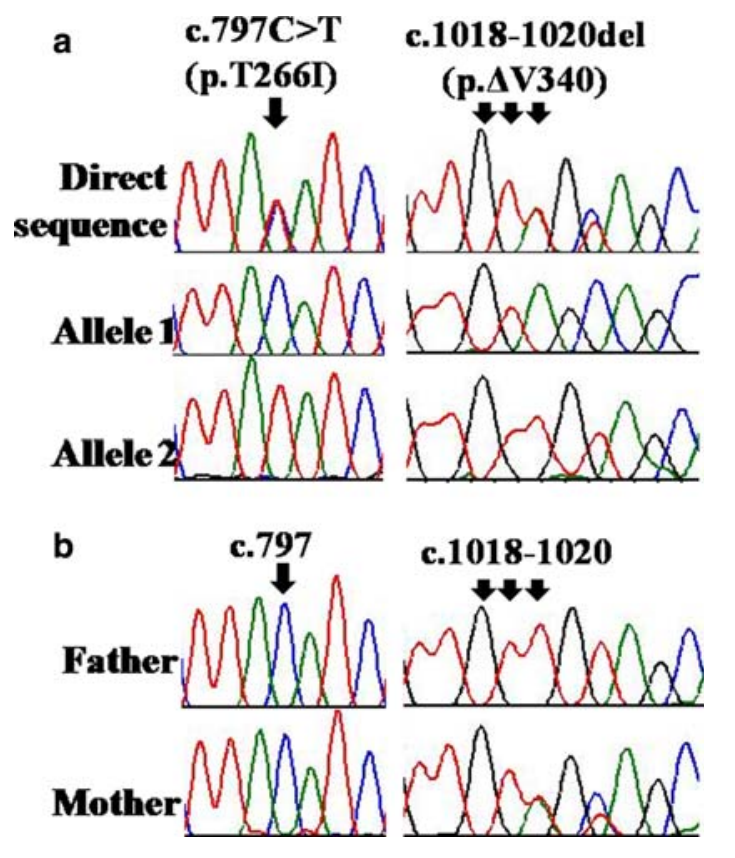

Fig. 3 a Sequences of the patient. Top direct sequence; middle and bottom sequences after TA cloning. The red, blue, green, and black waves represent nucleotides, thymine, cytosine, and adenine and guanine, respectively. The patient had heterozygous DTDST mutations, c.797C > T (p.T266I) and c.1018-1020del (p. $\Delta$ V340). b Direct sequences of the parents. The mother was a heterozygote of c.10181020del (p. $\Delta$ V340), but the father had neither of these two mutations

\section{Discussion}

r-MED and DTD are milder diseases in the DTD group. r-MED patients are not short, and they are healthy in childhood other than occasional associations with clubfeet and cleft palate. The radiological hallmarks include, as in the child in this study, broad proximal femora with proximal femoral epiphyseal dysplasia and undertubulated short tubular bones. By contrast, DTD presents with pre- and postnatal short stature, distinctive hitchhiker thumbs, inflammatory ear swelling, and intractable clubfeet, as well as joint dislocations and spinal malalignment. The skeletal changes are variable among affected individuals. Broad proximal femora and undertubulated short tubular bones are occasional, but not exclusive, findings. The presence of short stature and the radiological constellation of the child reported here fit an intermediate between r-MED and DTD.

Of note is that in this case, skeletal changes in infancy were reminiscent of Desbuquois dysplasia. r-MED and DTD also show broad proximal femora with prominence of the lesser trochanters, but those are not so prominent that are comparable to Swedish-key appearance. Mildly advanced carpal and tarsal ossifications are known in DTD but are not so prominent as those in this case or Desbuquois dysplasia.
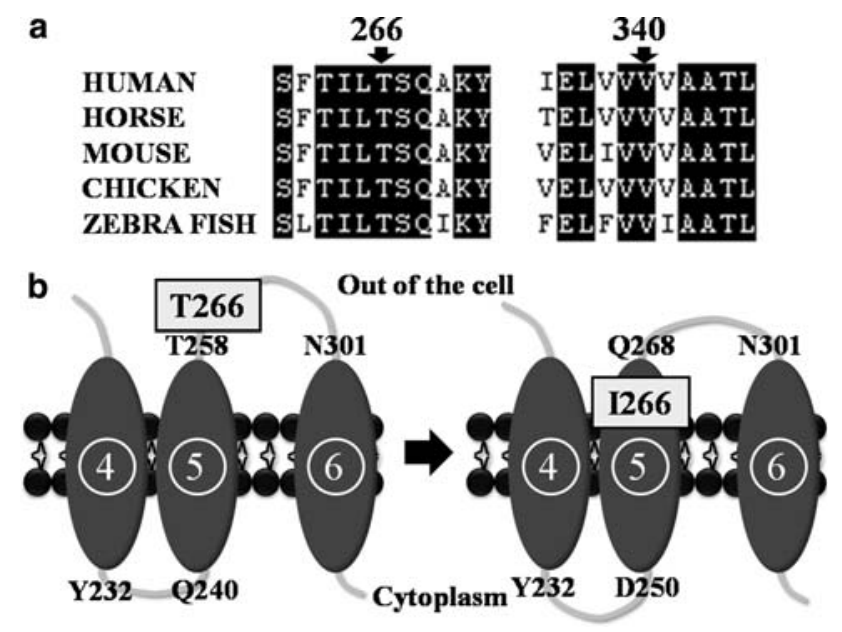

Fig. 4 Characterization of mutated amino acids. a Conservation of p.T266 and p.V340 in the diastrophic dysplasia sulfate transporter (DTDST) protein among different species. b Structural change of a DTDST protein predicted by PSORT. Ellipses represent the transmembrane domains, and curved lines represent either extracellular or cytoplasmic domains. The plain numbers are neighboring amino acid numbers of transmembrane domains shown at those positions. The circled numbers are transmembrane domain numbers from the $\mathrm{N}$-terminus. If a 266th amino acid (squared) changed from threonine $(T)$ to isoleucine $(I)$, it would migrate from outside of the cell into the plasma membrane. The length of the fifth transmembrane domain would not change, but that of the neighboring third extracellular and third cytoplasmic domain would be shortened and elongated by ten amino acids, respectively

c.1018-1020del (p. $\Delta$ V340) is a relatively common mutation in DTD and ACG-1B. Immunofluorescence analysis showed that p. $\Delta \mathrm{V} 340$ was not on the plasma membrane, although DTDST was a plasma membrane protein. Therefore, it was classified as a null mutation and associated with severe phenotypes of the DTD group (Karniski 2004). c.797C > T (p.T266I) is a novel mutation, which is supported by its de novo occurrence during paternal gametogenesis. Conservation between diverse species suggests that p.T266 plays an important role in DTDST protein. T266 was predicted to be outside of the plasma membrane because of its $\mathrm{OH}$ base, which had no affinity to lipid but to $\mathrm{H}_{2} \mathrm{O}$. On the other hand, I266 was predicted to migrate in the plasma membrane because it had nonpolarity and high affinity to lipid. As a consequence, the structure of DTDST protein would change drastically, most likely affecting its sulfate transport function.

According to a previous report (Karniski 2004), nonlethal disorders caused by DTDST mutations would be either a heterozygote of partial-function mutation and null mutation or a homozygote of partial-function mutations. The phenotypes of the child in this study fell into the milder range of the DTD group, and she had p. $\Delta \mathrm{V} 340$, a null mutation. Therefore, it seems that c.797C $>$ T (p.T266I) is classifiable as partial-function mutation, the sulfate transport activity of which is only mildly affected 
and in which localization is predicted to be on the plasma membrane. The result of the PSORT analysis supports this presumption. Further functional evidence is needed to validate the mutation function.

Thus, we first report a novel DTDST mutation, p.T266I, that results in a novel phenotype in combination with a recurrent null mutation, further extending the phenotypic spectrum of DTDST mutations. We hope that these findings will support clinical and genetic diagnosis of similar chondrodysplasias.

Acknowledgments We thank the patient and her parents for cooperation in the study. This work was supported by grant-in-aid from Development of New Approach for Regenerative Medicine.

\section{References}

Faivre L, Cormier-Daire V, Younf I, Bracq H, Finidori G, Padovani JP, Odent S, Lachman R, Munnich A, Maroteaux P, Le Merrer M (2004) Long-term outcome in Desbuquois dysplasia: a followup in four adult patients. Am J Med Genet A 124:54-59

Hall CM (2002) International nosology and classification of constitutional disorders of bone (2001). Am J Med Genet 113:65-77

Hastbacka J, de la Chapelle A, Mahtani MM, Clines G, Reeve-Daly MP, Daly M, Hamilton BA, Kusumi K, Trivedi B, Weaver A, Coloma A, Lovett M, Buckler A, Kaitila I, Lander ES (1994) The diastrophic dysplasia gene encodes a novel sulfate transporter: positional cloning by fine-structure linkage disequilibrium mapping. Cell 78:1073-1087

Hastbacka J, Superti-Furga A, Wilcox WR, Rimoin DL, Cohn DH, Lander ES (1996) Atelosteogenesis type II is caused by mutations in the diastrophic dysplasia sulfate-transporter gene (DTDST): evidence for a phenotypic series involving three chondrodysplasias. Am J Hum Genet 58:255-262

Ikeda T, Mabuchi A, Fukuda A, Hiraoka H, Kawakami A, Yamamoto S, Machida H, Takatori Y, Kawaguchi H, Nakamura K, Ikegawa $S$ (2001) Identification of sequence polymorphisms in two sulfation-related genes, PAPSS2 and SLC26A2, and an association analysis with knee osteoarthritis. J Hum Genet 46:538-543

Karniski LP (2001) Mutations in the diastrophic dysplasia sulfate transporter (DTDST) gene: correlation between sulfate transport activity and chondrodysplasia phenotype. Hum Mol Genet 10:1485-1490
Karniski LP (2004) Functional expression and cellular distribution of diastrophic dysplasia sulfate transporter (DTDST) gene mutations in HEK cells. Hum Mol Genet 13:2165-2171

Lachman RS (1998) International nomenclature and classification of the osteochondrodysplasias (1997). Pediatr Radiol 28:737-744

Maeda K, Miyamoto Y, Sawaki H, Karniski LP, Nakashima E, Nishimura G, Ikegawa S (2006) A compound heterozygote harboring novel and recurrent DTDST mutations with intermediate phenotype between Atelosteogenesis type II and Diastrophic dysplasia. Am J Med Genet A 140:1143-1147

Mégarbané A, Haddad FA, Haddad-Zebouni S, Achram M, Eich G, Le Merrer M, Superti-Furga A (1999) Homozygosity for a novel DTDST mutation in a child with a 'broad bone-platyspondylic' variant of diastrophic dysplasia. Clin Genet 56:71-76

Nishimura G, Hong HS, Kawame H, Sato S, Cai G, Ozono K (1999) A mild variant of Desbuquois dysplasia. Eur J Pediatr 158:479-483

Rossi A, Superti-Furga A (2001) Mutations in the diastrophic dysplasia sulfate transporter (DTDST) gene (SLC26A2): 22 novel mutations, mutation review, associated skeletal phenotypes, and diagnostic relevance. Hum Mutat 17:159-171

Rossi A, Bonaventure J, Delezoide AL, Superti-Furga A, Cetta G (1997) Undersulfation of cartilage proteoglycans ex vivo and increased contribution of amino acid sulfur to sulfation in vitro in McAlister dysplasia/atelosteogenesis type 2. Eur J Biochem 248:741-747

Rossi A, Kaitila I, Wilcox WR, Rimoin DL, Steinmann B, Cetta G, Superti-Furga A (1998) Proteoglycan sulfation in cartilage and cell cultures from patients with sulfate transporter chondrodysplasias: relationship to clinical severity and indication on the role of intracellular sulfate production. Matrix Biol 17:361-369

Superti-Furga A, Hastbacka J, Wilcox WR, Cohn DH, van der Harten HJ, Rossi A, Blau N, Rimoin DL, Steinmann B, Lander ES, Gitzelmann R (1996a) Achondrogenesis type IB is caused by mutations in the diastrophic dysplasia sulphate transporter gene. Nat Genet 12:100-102

Superti-Furga A, Rossi A, Steinmann B, Gitzelmann R (1996b) A chondrodysplasia family produced by mutations in the diastrophic dysplasia sulfate transporter gene: genotype/phenotype correlations. Am J Med Genet 63:144-147

Superti-Furga A, Neumann L, Riebel T, Eich G, Steinmann B, Spranger J, Kunze J (1999) Recessively inherited multiple epiphyseal dysplasia with normal stature, club foot, and double layered patella caused by a DTDST mutation. J Med Genet 36:621-624

Superti-Furga A, Unger $S$, the Nosology group of the international skeletal dysplasia society (2007) Nosology and classification of genetic skeletal disorders: 2006 revision. Am J Med Genet A 143:1-18 